\title{
PROSPECTS
}

\section{The right kind of risk}

\section{More young scientists should dedicate a portion of their attention to high-risk research despite the potential downside, argues Abraham Loeb.}

I find it alarming that today's young astrophysicists often invest their time conservatively in mainstream ideas that have already been extensively explored. There is a better path for doing science.

The tendency to play it safe is driven by peer pressure and job-market prospects - and sometimes encouraged by senior researchers. Past decades have seen the same phenomenon, but it is more prevalent today because scientists are increasingly pursuing projects in large groups with rigid research agendas and tight schedules that promote predictable goals. This trend towards conservative science is particularly unsettling given that so much of the Universe remains a mystery. And astrophysics is not a special case - scientists in many fields feel pressure to subscribe to the prevailing dogma.

Young researchers in particular should resist this trend and pursue innovative research. The window of opportunity in a career is short: although tenure should allow scientists to take more risks, most senior researchers get distracted by administration and fund-raising. Tenured professors often maintain a conservative profile that promotes old ideas. A change in attitude, supported by policy changes at our funding agencies, is crucial for the future health of astrophysics and other disciplines - and taking risks in moderation benefits young careers as well.

\section{Investing in research time}

Astrophysics has both safe and risky topics - I like to think of them as 'bonds' (low risk), 'stocks' (medium risk) and 'venture capital' (VC; high risk). The best approach for the fledgling researcher is to diversify his or her academic portfolio, always making sure to devote some of it to innovative projects with risky but potentially highly profitable returns.

The average research-time investment strategy for postdocs in astrophysics is $80 \%$ bonds, $15 \%$ stocks and 5\% VC. But I recommend instead 50\% bonds, 30\% stocks and $20 \%$ VC on average, with individual choices depending on circumstances some risky projects may require $100 \%$ of a scientist's research time.

'Future investments in astrophysics' shows present-day examples of topics that I consider bonds, stocks or VC. Each one includes components with varying degrees of risk.

But taking risks can be frustrating. Just as the majority of monetary VC investments result in loss, most risky projects end in failure and lost time. And such risky projects

\begin{tabular}{|lll|}
\hline FUTURE INVESTMENTS IN ASTROPHYSICS \\
\hline Bonds & Stocks & Venture capital \\
\hline Precision cosmology & $\begin{array}{l}\text { Time-dependent dark } \\
\text { energy }\end{array}$ & $\begin{array}{l}\text { Modified theories of } \\
\text { gravity }\end{array}$ \\
\hline $\begin{array}{l}\text { Standard-model } \\
\text { refinements }\end{array}$ & $\begin{array}{l}\text { Dark-matter search in } \\
\text { the Large Hadron Collider } \\
\text { and Milky Way halo }\end{array}$ & $\begin{array}{l}\text { Anthropic reasoning } \\
\text { (landscape) }\end{array}$ \\
$\begin{array}{l}\text { Star formation and } \\
\text { galaxy evolution }\end{array}$ & $\begin{array}{l}\text { Annihilation/decay } \\
\text { signatures of dark matter }\end{array}$ & $\begin{array}{l}\text { No Big Bang or } \\
\text { inflation }\end{array}$ \\
$\begin{array}{l}\text { Black-hole feedback and } \\
\text { growth in galactic nuclei }\end{array}$ & $\begin{array}{l}\text { Constraints on cosmic } \\
\text { inflation }\end{array}$ & $\begin{array}{l}\text { Variable constants of } \\
\text { nature }\end{array}$ \\
\hline
\end{tabular}

Research-investment trajectories in astrophysics

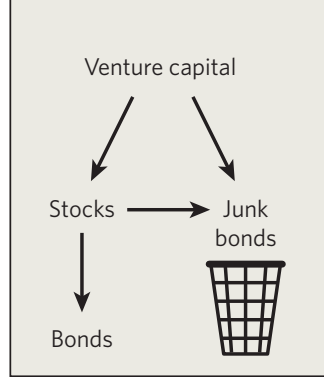

bring about isolation. Even after a truth is discovered, the rest of the community often remains silent. This loneliness is in contrast to the nurturing feedback that accompanies work on variations of existing, accepted themes.

Add to these trade-offs the growing proportion of projects that are done in large groups with rigid research agendas. In cosmology, the emergence of a 'standard model' - in which the composition, geometry, large-scale structure and expansion rate of the Universe are well constrained - offers secure research bonds in which young cosmologists can invest their time with minimal risk. Although it is possible to focus on more radical aspects of the model, most prefer not to be avant-garde. When I was a postdoc two decades ago, the lack of a prevailing paradigm made it more socially acceptable for young cosmologists to invest in VC ideas.

An individual's chosen level of risk is a mixture of personal inclination and social factors. And timing is crucial for making a profit. When should a researcher be inclined to invest in a bond, stock or VC? It depends on the state of the field. Consider the choice between dark matter and a modified theory of gravity to explain certain astronomical observations. If experiments reduce the upper limit on the cross-section of dark-matter particles below that of most reasonable theoretical models, alternative gravity models will seem more appealing.

But high-risk research, like a VC investment, has the potential to be more profitable than other investments. Even if only one in a million non-mainstream ideas bears fruit, it could transform our view of reality and justify the entire effort. And as long as young researchers maintain a balanced portfolio, high-risk work that fails will not doom young careers.

'Research-investment trajectories in astrophysics' illustrates the historical trajectories of ideas that started as VC, turned into stocks and, with better data and theoretical insights, eventually matured into bonds. Examples include the Big Bang, the cosmic microwave background and dark matter - all radical concepts that gained acceptance with the collection of empirical data.

But other ideas that started as VC have been abandoned owing to new observations. Examples include steady-state cosmology, which held that the Universe has existed forever without a Big Bang, and the notion that dark matter is made of ordinary matter.

\section{Fool's gold}

My advice should not be interpreted as encouragement to study every idea that comes along. I suggest that young scientists avoid ideas that are speculations on top of a speculation, such as postulating a modified theory of gravity, then adding the notion of an undetected form of matter (dark matter) to make that theory fit all existing data. And research priorities may need to be reallocated depending on what is discovered. Those who uncover an unexpected, intriguing result at the end of a project should consider making it the focus of their main research paper, despite the time invested elsewhere.

All this means that selection and promotion committees and grant-awarding agencies in all fields of science must find new ways to reward creative thinking. For example, funding agencies could allocate a fixed fraction of their budget to risky but potentially highly profitable projects outside the mainstream. We will all benefit from the implementation of a strategy that encourages innovation. And for those who take the road less travelled, keep your spirits up. After all, is there any point to doing science other than to take that road?

Abraham Loeb is director of the Institute for Theory and Computation at Harvard University in Cambridge, Massachusetts. This article is adapted from a June 2010 lecture (see go.nature.com/18Ydn5). 